\title{
Index of Authors
}

Adam S.: 15

Afonso C.L.: 175

Agaba M.: 89, 293

Ait-Ali T.: 239

Alencar M.M.: 225

Alexander L.J.: 231

Allan M.F.: 327

Amaral M.E.J.: 343, 359

Archibald A.L.: 89, 239, 407

Audonnet J.-C.: 407

Avendano S.: 373

Azevedo A.L.S.: 225

Babiuk L.: 407

Babu U.: 141

Bagnall N.: 77

Barris W.: 219

Barrow P.: 57

Bearson S.M.D.: 105

Beaumont C.: 353

Bed'Hom B.: 267, 271

Bellgard M.: 77

Bennett G.L.: 327

Bishop S.C.: 199, 239, 407

Boettcher D.: 301

Botti S.: 399

Brass A.: 89, 293

Bridle B.: 321

Burlot T.: 353

Burnside J.: 365

Burt D.: 57

Buschbell H.: 161

Caetano A.R.: 343

Cahaner A.: 153

Calenge F.: 353

Cantu M.: 399

Cardoso V.L.: 287

Cavanagh J.J.: 219
Chardon P.: 99

Chazara O.: 271

Chen K.: 15

Cheng H.: 365

Choudhary S.: 261

Cochet M.: 99

Costa A.: 399

Counter C.: 15

Coutinho L.L.: 225

Couture O.P.: 105

Crawford A.M.: 205

Crump R.E.: 219

Dekkers J.C.M.: 105, 213, 365

Diack A.B.: 255

Dodds K.G.: 205

Dodgson J.: 365

Doran T.J.: 377

Dowd S.E.: 115

Drew T.W.: 239

Eckersall P.D.: 255

Elsasser T.H.: 391

Fan B.: 337

Fife M.: 57

Fisher P.: 293

Flori L.: 99

Fravalo P.: 353

Freitas M.A.R.: 287

Frossard J.P.: 239

Fulton J.: 365

Galina-Pantoja L.: 35

Gasbarre L.C.: 231

Gasparin G.: 225

Gay C.G.:XIII, 3, 407

Genini S.: 399

Gheyas A.: 199
Gianola D.: 373

Gibson J.: 89

Gill C.A.: 343

Giuffra E.: 399

Gladney C.D.: 35, 255

Goddard M.E.: 383

Gourichon D.: 267

Guerrero F.D.: 115

Guillou M.:XV,

Gupta N.: 261

Gupta S.C.: 261

Guy D.R.: 199

Haley C.S.: 199

Hall L.: 293

Hamilton A.: 199

Hanotte O.: 89

Harhay G.P.: 169

Hasenstein J.R.: 213

Hassen A.T.: 213

Hawken R.J.: 219

He H.: 57

Heller E.D.: 153

Heriazon A.: 315

Hicks J.: 43

Hobbs M.: 219

Hong Y.H.: 67, 121, 131

Horin P.: 347

Houston R.D.: 199

Howell J.: 57

Hubertova D.: 347

Hugot K.: 99

Hulme H.: 89, 293

Hulst M.M.: 27

Hunt H.: 365

Ibelli A.M.G.: 225 
Jackson L.: 77

Jonas E.: 161, 279

Jones M.: 57

Jonsson N.: 77

Jozani R.J.: 321

Juengst H.: 161, 279

Kaiser P.: 57

Kapczynski D.R.: 175

Karrow N.A.: 247, 331

Kata S.: 359

Kataeva G.: 307

Kaushik A.K.: 307

Keane O.M.: 205

Keele J.W.: 231

Keeler Jr. C. L.: 121, 141

Kemp S.J.: 89, 293

Khatkar M.: 219

Kim C.-H.: 67, 121, 141

Kim D.K.131

Kim T.: 365

Kogut M.: 57

Kongsuwan K.: 77

Koohmaraie M.: 327

Koren E.: 153

Koti M.: 307

Kubickova S.: 347

Kuehn L.A.: 327

Kuhar D.: 105

Kuzmuk K.: 15

Lager K.M.: 169

Lamont S.J.: 131, 153, 213, 365

Lange A.: 93

Lecerf F.: 353

Lefèvre F.: 99

Lemonnier G.: 99

Lew A.: 77

Leyva-Baca I.: 247

Li C.-J.: 391

Li R.W.: 231, 391

Lillehoj H.S.: 67, 121, 131, 141

Liu G.E.: 231

Liu H.C.: 43

Lôbo R.B.: 287

Long N.: 373

Lowenthal J.W.: 377

Lunney J.K.: 105

Maak S.: 301

Machado M.A.: 225
MacLea K.S.: 365

Malik G.: 261

Malinverni R.: 399

Mallard B.A.: 315, 321, 407

Mao W.: 365

Mariani V.: 99

Marra D.: 399

Martins da Silva A.: 287

Matiasovic J.: 347

Matukumalli L.K.: 231

Mazur M.: 15

McAndrew B.J.: 199

McEwan J.C.: 205

McKay J.: 407

Mellencamp M.A.: 35, 239, 255

Miglior F.: 315

Miller L.C.:

Minozzi G.: 267

Minvielle F.: 267

Miyata M.: 225

Moolhuijzen P.: 77

Morgan R.: 365

Moser G.: 219

Mote B.: 337

Mount J.: 331

Muir W.: 365

Murani E.: 185

Musilova P.: 347

Nakata L.C.: 225

Necesankova M.: 347

Neill J.D.: 93, 169

Nene V.M.: 115

Nettleton D.: 105

Nicholas F.W.: 219

Niikura M.: 365

Nikkilae M.: 337

Nino-Soto M.I.: 315, 321

Noyes H.A.: 89, 293

Oliveira M.C.S.: 225

Onteru S.K.: 337

Osickova J.: 347

Ouyang M.: 365

Pandey A.: 261

Pant S.D.: 247

Pantin-Jackwood M.: 175

Park D.W.: 131

Parmentier H.K.: 267

Pastoret P.-P.:XIII
Phatsara C.: 161, 279

Pinard-Van der Laan M.-

H.:XIII, 3, 267, 271, 407

Piper E.K.: 77

Pitcovski J.: 153

Pitel F.: 353

Plastow G.: 407

Poh T.-Y.: 57

Pollock C.: 15

Ponsuksili S.: 161, 185, 279

Prevost K.: 353

Protais J.: 353

Qu L.: 105

Quinton M.: 315

Raadsma H.W.: 219

Ramos E.S.: 287

Rebel J.M.J.: 27

Regitano L.C.A.: 225

Rehfeldt C.: 301

Rennie C.: 89, 293

Ridpath J.F.: 93

Riggs P.K.: 343

Rios A.F.L.: 287

Robin S.: 99

Rodrigues Filho E.A.: 343

Rogatcheva R.: 15

Rogel-Gaillard C.: 99

Rosa G.J.M.: 373

Rothschild M.F.: 337

Rothwell L.: 57

Rubes J.: 347

Rund L.: 15

Ryan K.: 77

Sadeyen J.-R.: 57

Saldivar L.: 115

Salmon N.: 57

Sarmento L.: 175

Schafer D.S.: 377

Schellander K.: 161, 279

Schenkel F.S.: 247

Schmidt R.: 301

Schook L.B.: 15

Schreinemachers H.-J.: 279

Sellier N.: 353

Serenius T.: 337

Sharma B.S.: 247, 331

Silva A.M.: 225

Silva M.V.G.B.: 225 
Smith J.: 57

Smith T.L.: 169

Smith T.P.: 231

Smits M.A.: 27

Sölkner J.: 219

Soller M.: 365

Sonstegard T.S.: 225, 231

Stafuzza N.B.: 343, 359

Stalder K. J.: 337

Stear M.J.: 255

Stevens M.: 57

Swaggerty C.: 57

Swalve H.H.: 301

Swayne D.E.: 175

Taggart J.B.: 199

te Pas M.F.W.: 27

Teodoro R.L.: 225

Tesfaye D.: 161, 279

Thaller G.: 301

Thallman R.M.: 327

Tholen E.: 161, 279

Thompson K.A.: 315

Tier B.: 219

Tinch A.: 199

Tixier-Boichard M.: 271

Torremorell M. : 35, 407

Tuggle C.K.: 105

Uthe J.J.: 105

Vallat B.: 429

Van Diemen P.: 57

Van Tassell C.P.: 231

Velge P.: 353

Vercesi Filho A.E.: 287

Vigna A.: 353

Vyskocil M.: 347

Wang M.: 115

Wang Y.F.: 105

Weigel K.A.: 373

Westcott D.G.: 239

Wigley P.: 57

Wilson A.D.: 239

Wimmers K.: 161, 185, 279

Wise T.G.: 377

Womack J.E.: 343, 359
Yammuen-art S.: 161

Yoo D.: 43

Young J.: 57

Zaros L.G.: 225

Zenger K.: 219

Zhang M.: 141

Zhang S.: 141

Zhou H.: 153

Zuerner R.L.: 93 